\title{
BMJ Open Medication adherence, self-efficacy and health literacy among patients with glaucoma: a mixed-methods study protocol
}

\author{
Maria Achilleos (D) , ${ }^{1}$ Anastasios Merkouris, ${ }^{1}$ Andreas Charalambous (D) , ${ }^{1,2}$ \\ Evridiki Papastavrou ${ }^{1}$
}

To cite: Achilleos M, Merkouris A, Charalambous A, et al. Medication adherence, self-efficacy and health literacy among patients with glaucoma: a mixed-methods study protocol. BMJ Open 2021;11:e039788. doi:10.1136/ bmjopen-2020-039788

- Prepublication history for this paper is available online. To view these files, please visit the journal online (http://dx.doi org/10.1136/bmjopen-2020039788).

Received 02 May 2020 Revised 29 December 2020 Accepted 09 January 2021

Check for updates

(c) Author(s) (or their employer(s)) 2021. Re-use permitted under CC BY-NC. No commercial re-use. See rights and permissions. Published by BMJ.

${ }^{1}$ Nursing, Faculty of Health Sciences, Cyprus University of Technology, Limassol, Cyprus ${ }^{2}$ Nursing, Turun Yliopisto, Turku, Finland

Correspondence to Mrs Maria Achilleos; maria_ach_@hotmail.com

\section{ABSTRACT}

Introduction As the world population ages, glaucoma is becoming an increasingly significant cause of blindness. A key component in the management of glaucoma is the use of prescribed medications and the adherence to treatment. However, there is evidence of low adherence to prescribed medication in chronic diseases, such as glaucoma. This study aims to explore the level of medication adherence, self-efficacy, social support and health literacy among the patients with glaucoma and to determine if there are any correlations between them. The ultimate aim is to use the information to develop an educational programme for patients with glaucoma at a later stage.

Methods and analysis This is a mixed-methods study which includes two stages: a descriptive study (stage 1) and focus group discussions (stage 2). Sample: Patients with glaucoma or ocular hypertension, using at least one kind of drops, from two ophthalmology clinics. Selected measures include: The Glaucoma Treatment Compliance Assessment Tool, The European Health Literacy Survey Questionnaire, The Glaucoma Medication Self-Efficacy Questionnaire and The Multidimensional Scale of Perceived Social Support. Two focus groups will be used for the collection of qualitative data, aiming to enrich the study with the patients' experiences. The data will be analysed with SPSS, using descriptive and inferential statistics for stage 1 whereas content analysis will be used for the data from the focus group discussions (stage 2).

Ethics and dissemination Permission to conduct the study was received from the National Bioethics Committee and the board of management of the two ophthalmology clinics. All participants will be informed fully on the purpose and methods of the study. Consent forms will be signed and at any time participants will have the right to withdraw. Confidentiality and the protection of data will be respected at all times.

\section{INTRODUCTION}

As the world population ages, glaucoma is becoming an increasingly significant cause of blindness. ${ }^{1-3}$ The global prevalence of glaucoma for population aged 40-80 years is $3.54 \%,{ }^{4}$ while Quigley and Broman, ${ }^{2}$ previously estimated that proportion to be $2.65 \%$. However, the problem could be much greater strengths and limitations of this study

The main strength of the study lies in the use of two different methodological approaches that will give a better understanding of the problem under study.

- The study is going to be conducted only in two ophthalmology clinics, however, the two clinics together accept the majority of glaucoma patients in the country.

- The sample size of the study is around $10 \%$ of the known population with glaucoma in the current country.

- We have chosen to use self-reporting instruments to assess adherence instead of monitoring devices, and that may overestimate adherence, however, there is no gold standard to assess adherence; both methods have their limitations.

- As far as we know, this is the first study aiming to determine if there are any correlations between medication adherence, self-efficacy, health literacy and social support.

since there is no mandatory case reporting system for glaucoma (such as the cancer registry). International statistics by $\mathrm{WHO}^{3}$ show that glaucoma is the second leading cause of blindness (8\%), worldwide, after cataracts $(51 \%)$. Despite this, glaucoma presents a greater public health challenge since it will lead to irreversible blindness if left untreated or if the patient is not adherent to the treatment. ${ }^{156}$

The pathophysiology, presentation and treatment of glaucoma cannot be explained using a single definition due to the different types of glaucoma that exist. Generally, glaucoma is a chronic disease that 'exhibits a characteristic optic neuropathy which may result in progressive visual field loss ${ }^{6}$ In essence, the most important risk factor for glaucoma is raised intraocular pressure (IOP). When the IOP is high for a long time, the optic nerve fibre is progressively damaged, with structural 
changes and functional deficits. ${ }^{5}$ Usually, defects in the visual field are asymptomatic at the beginning, but the peripheral vision is gradually affected and the patient observes a tunnel vision. This leads to delayed diagnosis and as a result to unchangeable visual field defects. The most acceptable intervention to prevent further loss of vision and control glaucoma is lowering IOP to a safe level for the eye. ${ }^{56}$ The recommended steps to achieve IOP reduction are mainly topical medications followed by more invasive methods (such as laser or surgeries). In order to have a successful treatment, patients have to monitor for life and follow their doctor's medication plan with full consistency.

Although glaucoma is a sight-threatening condition, and it is important that it is treated early, there is evidence that around 26,5\% of glaucoma patients seem to be non-adherent to treatment. ${ }^{7-10}$ The European Glaucoma Society has given a wider range of non-adherence, between $30 \%$ and $70 \% .^{5}$ It is worth mentioning that patients are considered adherent if they use $80 \%$ or more of the prescribed doses, and they classify as nonadherent or low adherent if they use less than $80 \%$ of the prescribed doses as suggested in prior research. ${ }^{11-14}$ Based on the above, at least one patient out of four is less than $80 \%$ adherent to the treatment. According to WHO in 2020, 76 million people from 40 to 80 years of age have glaucoma. ${ }^{15}$ This means that approximately 19 million people are non-adherent to their treatment and this is a very crucial finding since the lack of adherence to glaucoma therapy is closely related to the progression of glaucoma. ${ }^{5} 121617$

Glaucoma, related to blindness, represents a substantial economic and psychological burden for the patient and the society as a whole. ${ }^{141617}$ To be more specific, the prevalence of glaucoma contributes to significant direct and indirect $\operatorname{costs}^{18-21}$; whereas the annual cost per patient treatment increases as the severity of the disease worsens. ${ }^{18}$ As the condition progresses, patients are less able to perform their daily activities and significant physical challenges start to appear. When glaucoma patients were compared with a healthy control group, it was found that they were over three times more likely to have had a fall in the previous 12 months and five times more likely to have been involved in a motor vehicle collision..$^{22}$ On the other hand, the psychological burden also increases as vision decreases. The impact of visual field loss on the patient's Quality of Life (QoL) is linear with greater visual field loss associated with a worse QoL. ${ }^{23}$ Undoubtedly, individuals with glaucoma were at somewhat higher risk for depression, fractures, nursing home admissions and home healthcare service use, ${ }^{2425}$ while their families are exposed to an increased psychological burden. ${ }^{26}$ Early diagnosis and appropriate therapy can prevent lifelong disability and preserve the patient's QoL. ${ }^{5}$

Consequently, all glaucoma patients require a careful monitoring by a specialist and a lifetime adherence to their treatment. Even so, almost one-fourth of the patients with glaucoma seem to be non-adherent to their treatment. Every patient is different and there are several types of non-adherence. Failure to take the correct medication as prescribed due to underdosing or overdosing, wrong medication, wrong timing of dosages, incorrect self-administration, forgetfulness, side effects, issue of cost or missed refills are some of the reasons that may affect adherence. ${ }^{5727}$ The researchers made an effort to classify all these reasons into thematic maps. This led them to the conclusion that health literacy, self-efficacy and social support can explain the majority of these reasons. Taking into account that, a scoping review was conducted in order to synthesise research evidence and provide an overview of the possible relations between glaucoma medication adherence, self-efficacy, health literacy and social support. The results of this review were collected, summarised and reported in themes below.

\section{Medication adherence and health literacy}

Research has shown that patients with low literacy tend to self-manage chronic diseases poorly. ${ }^{28-30}$ However, the mechanisms connecting health literacy and glaucoma management are not clear, so further research is needed to help researchers identify the relation between health literacy and medication adherence. Currently, there is evidence to show that low health literacy skills may be associated with low adherence to the prescribed glaucoma medication, ${ }^{31-34}$ as well as that patients with decreased health literacy skills demonstrate more advanced visual field loss. ${ }^{33}$ A cross-sectional survey, for example, found a positive correlation between health literacy and the number of refills obtained $(p=0.003),{ }^{31}$ whereas an educational intervention study with the goal of improving glaucoma medication adherence by targeting health literacy levels, showed that medication adherence can be improved in less literate patients through literacylevel appropriate education. ${ }^{32}$ Even though the results are encouraging, further research is needed concerning the way health literacy affects the adherence to glaucoma medications.

\section{Medication adherence and self-efficacy}

Another variable that can affect medication adherence, is self-efficacy. Glaucoma medications are administered via eye-drop bottles, and as a result, patients need to carry out specific tasks in order to achieve a desired outcome: they need (1) confidence to adhere and (2) confidence to administer their eye-drops correctly. ${ }^{35}$ There is evidence to show that patients with higher glaucoma medication self-efficacy seem to be significantly more adherent. ${ }^{76-39}$ But what can actually increase the self-efficacy of a glaucoma patient? According to the literature, patients who received education about glaucoma topics and expressed their personal views about glaucoma and its treatment, reported higher medication self-efficacy and significant increase in their confidence to overcome adherencerelated barriers. ${ }^{7}{ }^{37-39}$ Consequently, it is extremely important to educate patients about glaucoma and assess their views about it. The health professionals must keep 
in mind that patients who ask more questions about their glaucoma medications may be less confident in using them. ${ }^{37}$ Interventions should be designed to increase patient medication self-efficacy, teach patients proper eye-drop techniques and address issues that affect adherence, such as forgetfulness or medication schedule.

\section{Medication adherence and social support}

Although to date, the relationship between glaucoma medication adherence and social support have yet to be fully assessed, Salman et al, found no significant associations between social support and adherence to glaucoma medications ${ }^{40}$ while Cook et al, found that the social support was a significant predictor of self-reported medication adherence in univariate analysis (but nonsignificant in the multiple Poisson regression model) ${ }^{36}$ On the other hand, a significant relationship between the two variables appears to exist as is evident from the large number of studies on other chronic diseases. ${ }^{41-45}$ Among other findings, social support was significantly associated with higher levels of medication adherence, ${ }^{42-45}$ was observed to reduce negative perceptions about medications, ${ }^{41}$ found to be associated with greater asthma control and ${ }^{41} \mathrm{QoL}$ and had a positive correlation with treatment self-efficacy $(\mathrm{p}<0.01) .{ }^{42}{ }^{46}$ It is also supported by the literature that patients who receive information and emotional support benefit by displaying good adherence and collaboration in their care. ${ }^{47}$ Nevertheless, evidence from the field of rationing research shows that patient education and support are the most often-missed types of nursing care worldwide. ${ }^{48} 49$ Therefore, one of the ultimate aims of this study is the preparation of an educational programme, which can be a useful tool for nurses in educating and supporting patients with glaucoma on how to manage their disease.

In conclusion, glaucoma is becoming an increasingly significant cause of blindness. A key component in the management of glaucoma is the use of prescribed medications and the adherence to treatment. However, there is evidence of low medication adherence in patients with glaucoma and many factors seem be involved. This study aims to explore the level of medication adherence, selfefficacy, social support and health literacy among the patients with glaucoma and to determine if there are any correlations between them.

\section{METHOD AND ANALYSIS \\ Review of evidence}

In order to synthesise research evidence, we used a scoping review. The main purpose was to examine the extent and the range of research activity, to summarise and disseminate research findings and finally to identify research gaps in the existing literature. ${ }^{50}$ The search was done on the 25th of March 2020, in three electronic databases: MEDLINE (and MEDLINE complete), CINAHL Plus and APA PsycINFO with the keywords: 'medication adherence' OR 'medication compliance' OR 'medication
Searches of MEDLINE (and MEDLINE complete), CINAHL Plus and APA PsycINFO on the $25^{\text {th }}$ of March 2020 with the keywords: 'medication adherence' $O R$ 'medication compliance' $O R$ 'medication concordance' OR 'medication persistence', glaucoma OR 'ocular hypertension', 'self-efficacy' OR confidence, 'health literacy', 'social support' OR 'family support' OR 'support network' generated 61 articles:

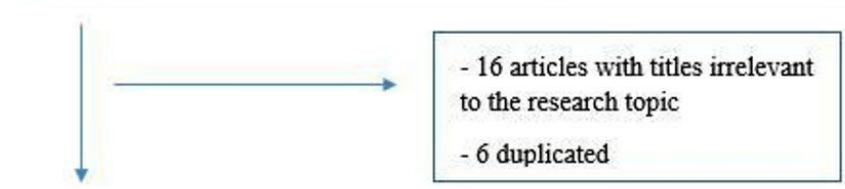

39 articles

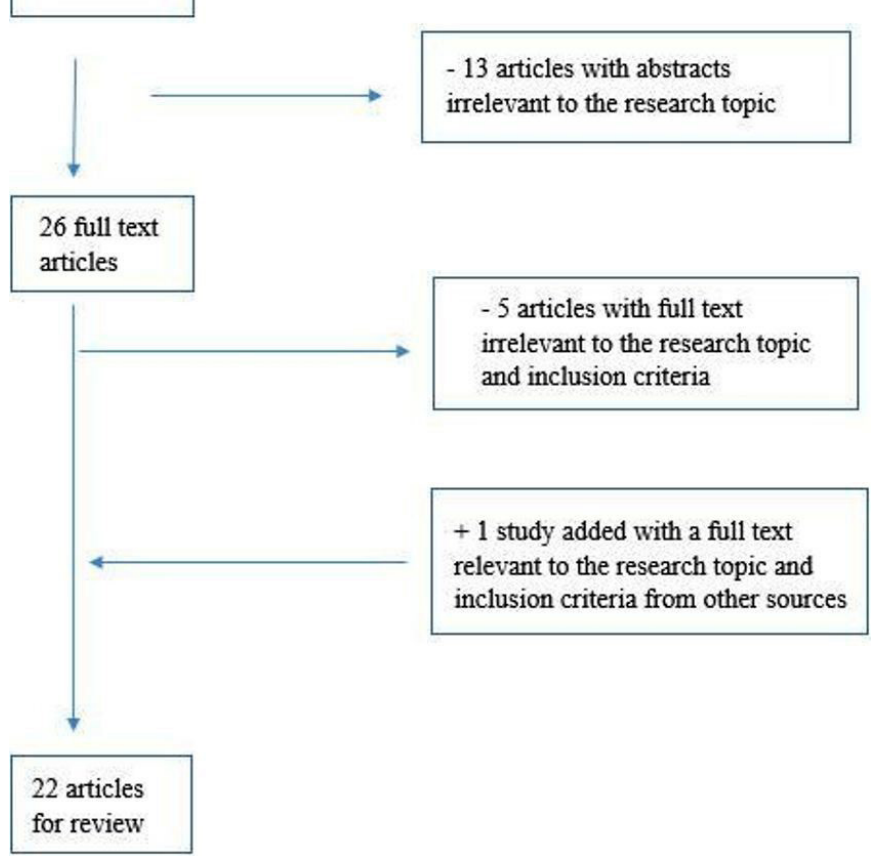

Figure 1 Flow diagram of search strategy. The flow diagram maps out the number of records identified, included and excluded, and the reasons for exclusions.

concordance' OR 'medication persistence', glaucoma OR 'ocular hypertension', 'self-efficacy' OR 'confidence', 'health literacy', 'social support' OR 'family support' OR 'support network'. Only studies in English were considered. No restrictions were set for research design or year of publication. The results were previously reported in themes whereas the search strategy is illustrated in figure 1 .

\section{Aim}

The aim of the study is to investigate the level of medication adherence, self-efficacy, social support and health literacy among the patients with glaucoma and to determine if there are any correlations between them. Also, we will try to enrich our results with patients' views as far as the possible reasons of low medication adherence. We may use the results of this study for the development of an educational programme for patients with glaucoma in the future.

The main research questions are: 
1. What is the level of medication adherence among patients with glaucoma?

2. What is the level of health literacy, self-efficacy and social support among patients with glaucoma?

3 . Is there any correlation between medication adherence and health literacy, self-efficacy or social support?

4. Is there any correlation between self-efficacy and health literacy or social support?

5. How do patients with glaucoma explain low adherence and what are the possible reasons for that?

\section{Design}

This is a mixed-methods study with an explanatory sequential design, consisting of two stages. First, a descriptive study will take place to evaluate the level of medication adherence, self-efficacy, social support and health literacy in a large population of patients with glaucoma, through the use of structured questionnaires (stage 1). A qualitative research with focus group discussions will follow (stage 2), in order to help us explain the quantitative data by investigating the patients' perceptions of their glaucoma and the problems they are facing in following the treatment.

\section{Stage 1: exploration of the level of medication adherence, self-efficacy, social support and health literacy in a large population}

In this stage, four structured questionnaires were consistent with our research questions: (1) The Glaucoma Treatment Compliance Assessment Tool (GTCAT) to assess the level of medication adherence, (2) The European Health Literacy Survey Questionnaire (HLSEU-Q6) to assess the health literacy among the patients, (3) The Glaucoma Medication Self-Efficacy Questionnaire (GMSEQ), to assess self-efficacy and (4) The Multidimensional Scale of Perceived Social Support (MSPSS) to assess the patient's social support. All the instruments were used in previous studies supporting their validity and reliability. ${ }^{351-57}$

\section{The Glaucoma Treatment Compliance Assessment Tool}

The GTCAT is an easy and quick questionnaire specially designed for patients with glaucoma. It contains 27 statements with 5-interval Likert-type scale response (eg, 1- disagree a lot, 5-agree a lot) and 1 open-ended question. The GTCAT assess the knowledge, the Health Belief Model (HBM) components (barriers, benefits, cues to action, self-efficacy, severity, susceptibility), the patientphysician relationship, and the patient's physical and mental health. The correct answers range from 0 to 27 , with higher scores indicating greater adherence to the treatment. The GTCAT was developed by Mansberger et $a \bar{l}^{58}$ according to (1) the constructs of the HBM, (2) expert opinion and (3) previous studies regarding adherence in patients with glaucoma. It has been translated to other languages and it is a valid and reliable tool (Cronbach's alpha >0.7). ${ }^{3653545859}$

\section{European Health Literacy Survey Questionnaire}

The HLS-EU-Q6 is the shortest version of the HLSEU-Q47 which was developed and validated by the HLS-EU consortium among eight European countries: Austria, Bulgaria, Germany, Greece, Ireland, the Netherlands, Poland and Spain ( $\mathrm{n}=1000$ per country, $\mathrm{n}=8000$ total sample). The HLS-EU-Q6 includes 6 statements with 5-interval Likert-type scale response (eg, 1- disagree a lot, 5-agree a lot) and was translated into Greek by the researchers after the Greek team attended the workgroup meetings for the development of the HLS-EU tool. ${ }^{56061}$ The HLS-EU-Q6 shows strong correlation with the full instrument, and researchers recommend the use of the shortest version of the instrument if the health literacy is not the primary variable (Cronbach's Alpha $=0.8) .^{55}$

\section{Glaucoma Medication Self-Efficacy Questionnaire}

To our knowledge, the Glaucoma Medication Self- Efficacy questionnaire is the only instrument specially designed to assess self-efficacy among patients with glaucoma. It consists of 10 questions assessing self-efficacy in glaucoma medication in general and six questions assessing self-efficacy in the eye-drop technique. The response categories are: 'not at all confident', 'somewhat confident', 'very confident' and 'does not apply'. It is important to mention that neither 'The eye-drop technique Self-Efficacy scale' was significantly associated with medication adherence, nor the 'Glaucoma Medication Adherence Self-Efficacy Scale' was significantly associated with any of the eye-drop technique measures. These findings are significant because they prove that the scales are measuring different aspects of glaucoma medication use (Cronbach's alpha $=0.91) .{ }^{35} 57$

\section{The Multidimensional Scale of Perceived Social Support}

The MSPSS was developed by Zimet et al. ${ }^{62}$ It has been widely used since then and has been translated and validated in various cultures. ${ }^{63-70}$ The MSPSS consists of twelve items and three subscales: (1) significant other subscale, (2) family subscale and (3) friends subscale. Each item is scored on a likert scale ranging from 1 (very strongly disagree) to 7 (very strongly agree). Any mean scale score ranging from 1 to 2.9 would be considered low support, a score of 3-5 would be considered moderate support and a score from 5.1 to 7 would be considered high support. The MSPSS is a short and easy to understand scale, suitable for populations with limited literacy level (Cronbach's alpha $=0.93) .{ }^{52}$

\section{Cultural adaptation of the research instruments}

The English versions of the GTCAT and the GMSEQ will be translated to Greek, using standard techniques, such as the forward-back translation. ${ }^{71-74}$ A pilot phase of the study will follow, where the prefinal Greek versions of the GTCAT and the GMSEQ and the already translated HLS-EU and MSPSS will be pilot tested among a sample of 30 participants. ${ }^{756}$ The target population will include all glaucoma patients, using at least one kind of drops 
and visiting the private ophthalmology clinic during the recruitment process. Those 30 patients will be included only in the pilot study and not in the main study.

The validation of the instruments will include face validity, content validity (Content Validity Index) and factor analysis. More specifically, both methods of factor analysis: confirmatory and explanatory, are going to be used. First, confirmatory factor analysis will aim to validate the structure of the instruments, showing whether the structure of the new, translated versions is the same as the factor structure of the original instruments. Exploratory factor analysis will additionally examine the structure of the translated instruments, showing if any items should be removed/moved to other factors. The reliability will be checked with Cronbach's alpha method. This deemed necessary (especially for comparison purposes) since all the related articles that have used these instruments documented the values of Cronbach's alpha.

Analysis will be performed by SPSS V.24.0. Medication adherence will be the main variable while health literacy, self-efficacy and social support will be the secondary variables. Higher total scores on the questionnaires will indicate higher medication adherence, health literacy, self-efficacy and social support, respectively. Descriptive statistics, such as means, SD, medians or percentages, will be reported and correlations between the variables will be explored with parametric and non-parametric measures.

The main scale in the study is GTCAT and this will be used as the dependent variable in all the regression analyses that will be performed, in an effort to examine which factors affect the level of medication adherence. Therefore, linear regression models (multiple regression models) are suitable for our analysis, due to the fact that the dependent variable is numerical (continuous). The usual assumptions of linear regression will be examined (eg, normality, homoscedasticity, etc), as far as hierarchical regression, to examine the effects of the other factors (scales), adjusting for sociodemographic characteristics.

\section{The sample}

The sample will include glaucoma patients from two ophthalmology clinics in Cyprus (one private and one public). To achieve 95\% CI with an acceptable error of $5 \%$, the sample size calculator, Raosoft, revealed sample estimation at 263 participants. In order to accommodate for any missing or invalid questionnaires an additional $10 \%$ is necessary. As a result, our study will approach 289 participants. Similarly, other references on structural equation modelling (SEM), which includes Confirmatory Factor analysis, indicate a ratio of 10 responses per free parameters to obtain trustworthy estimates ${ }^{77}$ or 10 participants per item in scale development. ${ }^{78}$ Based on the questionnaires that we have in the current study and given the above guidelines, a sample size close to 300 will be sufficient enough for all the scales in our study (since the largest scale in our study includes 27 items).

The inclusion criteria are:
- Patients older than 18 years of age.

- Ability to read and understand Greek.

- Diagnosis of glaucoma or ocular hypertension, requiring treatment with hypotensive eye-drops (at least once per day).

\section{The procedure}

The $\mathrm{PhD}$ candidate will visit both clinics and ask the physicians to identify patients with glaucoma (during their prescreening process) who meet the inclusion criteria and inform them about the study. Patients who are willing to participate will be referred to the researcher for an informed consent and to complete the questionnaires, including a section for the demographic data. Due to the patients' problem of vision, the questionnaires will be completed with the researcher's help who will read out the questions, without encouraging the answers. This is the main reason that only one researcher, the $\mathrm{PhD}$ candidate, will be responsible for the collection of data.

\section{Stage 2: focus group discussions}

The collection of data through focus group discussions, after the completion of the stage 1 , will allow us to examine in depth the patients' experiences of the daily use of glaucoma drops. The aim is to enrich the future educational programme with the specific needs of patients with glaucoma, since, the focus group discussions are invaluable for guiding the development of interventions and at the same time ensuring that these interventions meet patient needs ${ }^{79}$ as expressed by them.

Purposive sampling will be employed to ensure a range of patient experiences with medication adherence is assessed. The patients will differ in terms of the number of years using eye-drops to stimulate interaction between them and explore different views of the investigated topic in order to provide enriched data. ${ }^{80}$

Patients will be recruited to participate in two focus groups, from patient lists provided by the ophthalmology clinic. Each group will consist of 7-10 participants ${ }^{7981}$ and the duration of the meeting will not exceed 1 hour. The inclusion criteria will be the same as the first stage of the study with an additional parameter whereby the participants will be informed that they will be audiorecorded (but will keep their anonymity) and will sign again an informed consent before the beginning of the study. In order to provide a comfortable setting and eliminate the impact of the clinic's environment, the participants will be invited to attend the focus groups in a meeting room on the University campus.

The focus groups will be conducted by one researcher who will act as a moderator. The moderator will not be known to the participants, in order to reduce social desirability bias and "contamination of the data" ${ }^{82}$ The discussion will be audiorecorded and the non-verbal responses and interactions among the group members will be observed and written down by an independent observer. At the end of the session, the participants will be asked to fill in a questionnaire with demographic information. 


\section{Stage 1}

January -

September 2021

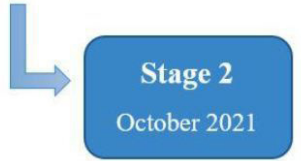

- Focus group discussions aiming to stimulate interaction between participants and share opinions about glaucoma treatment and adherence

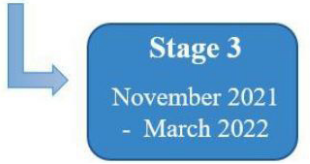

- Doctoral Dissertation writing

- Dissemination of findings

Figure 2 Timeline of the study. The descriptive study will begin in January 2021 and is expected to be completed in September 2021. A qualitative research with focus group discussions will follow in October 2021. Finally, the writing of the dissertation and the dissemination of findings will be completed by March 2022.

The focus group interviews will be tape-recorded and transcribed by the researcher and the analysis will be done by two investigators following a simple content analysis methodology. ${ }^{83}$

\section{Integration of the data}

This is a mixed-methods study with an explanatory sequential design, consisting of two stages; 'in sequential designs, the intent is to have one phase of the mixed-methods study build on the other'. ${ }^{84}$ At first, the researcher will collect and analyse the quantitative data, and then will inform the findings with qualitative data. ${ }^{85}$ The qualitative data from the focus group discussions can be used to assess the validity of the quantitative findings. According to Fetters et al, 'several advantages can accrue from integrating the two forms of data', hence, we assume that both stages will provide important insights into medication adherence and will help us clarify the possible reasons for low adherence, in relation to the patients' feelings and experiences about their glaucoma management and treatment. The timeline of the study is illustrated in figure 2 .

\section{Patient and public involvement}

During the both stages of the study, patients and public involvement will be of high importance. If the patients are not willing to participate, the study design will not be achievable.

\section{ETHICAL CONSIDERATION AND DISSEMINATION}

Permissions to conduct the study were obtained from the National Bioethics Committee and the board of management of the private and public clinics from where we will recruit the sample. Additionally, the Commissioner Bureau of Privacy Protection will be notified. All participants will be informed fully on the purpose and methods of the study. Consent forms will be signed and at any time participants will have the right to withdraw. Confidentiality of the participants will be respected. Researchers will safeguard the well-being of the participants during the data collection. Data from the two stages of the study will be stored in the researcher's office, where no one else is allowed to entry. The PhD candidate will be responsible for collecting, analysing, coding and recording the data; however, the supervising committee will monitor the whole procedure. The research team will be the only ones who will have access to the data (through the $\mathrm{PhD}$ candidate). If any important modification of the protocol takes place, all the relevant parties will be informed (ethics committee, health ministry). Dissemination strategy includes presentations in international and national scientific conferences. All the results of the study will be submitted to scientific journals for publication.

Twitter Andreas Charalambous @AndreasC466

Acknowledgements The authors would like to thank, in advance, all the patients for their cooperation and the departmental officers of the two clinics for their permission to conduct the descriptive stage of the study in their area.

Contributors MA as the first author, has written the manuscript. EP as the main supervisor of the project, coordinated and supervised the writing process. AM and $\mathrm{AC}$ have reviewed and contributed in writing the manuscript.

Funding The research is supported by the Cyprus Nursing and Midwifery Council grant number Y.Y.ГN.7813/AAE.5636 and Leventis foundation grant number 17 600. Publication fees were granted by the Cyprus University of Technology Open Access Author Fund.

Competing interests None declared.

Patient and public involvement Patients and/or the public were involved in the design, or conduct, or reporting, or dissemination plans of this research. Refer to the Methods section for further details.

Patient consent for publication Not required.

Ethics approval The protocol was approved by the Cyprus National Bioethics committee (EEBK EП 2019.01.220).

Provenance and peer review Not commissioned; externally peer reviewed.

Open access This is an open access article distributed in accordance with the Creative Commons Attribution Non Commercial (CC BY-NC 4.0) license, which permits others to distribute, remix, adapt, build upon this work non-commercially, and license their derivative works on different terms, provided the original work is properly cited, appropriate credit is given, any changes made indicated, and the use is non-commercial. See: http://creativecommons.org/licenses/by-nc/4.0/.

\section{ORCID iDs}

Maria Achilleos http://orcid.org/0000-0003-2235-9850

Andreas Charalambous http://orcid.org/0000-0003-4050-031X

\section{REFERENCES}

1 Kingman S. Glaucoma is second leading cause of blindness globally. Bull World Health Organ 2004;82:887-8.

2 Quigley HA, Broman AT. The number of people with glaucoma worldwide in 2010 and 2020. Br J Ophthalmol 2006;90:262-7.

3 WHO. Global data on visual impairment 2010, 2010.

$4 \mathrm{LiX}$, Tham Y-C, Cheng C-Y. Global prevalence of glaucoma and projections of glaucoma burden through 2040. Ophthalmology 2014;121:2081-90.

5 European Glaucoma Society. Terminology guidelines. 4th ed, 2014.

6 Kanski JJ. Clinical ophthalmology: a systematic approach. Sixth ed. Edinburgh: Butterworth-Heinemann/Elsevier, 2007.

7 Newman-Casey PA, Robin AL, Blachley T, et al. The most common barriers to glaucoma medication adherence: a cross-sectional survey. Ophthalmology 2015;122:1308-16.

8 Schwartz GF, Quigley HA. Adherence and persistence with glaucoma therapy. Surv Ophthalmol 2008;53:S57-68.

9 Olthoff CMG, Hoevenaars JGMM, van den Borne BW, et al. Prevalence and determinants of non-adherence to topical 
hypotensive treatment in Dutch glaucoma patients. Graefes Arch Clin Exp Ophthalmol 2009;247:235-43.

10 Olthoff CMG, Schouten JSAG, van de Borne BW, et al. Noncompliance with ocular hypotensive treatment in patients with glaucoma or ocular hypertension an evidence-based review. Ophthalmology 2005;112:953-61.

11 Martin BC, Wiley-Exley EK, Richards S, et al. Contrasting measures of adherence with simple drug use, medication switching, and therapeutic duplication. Ann Pharmacother 2009;43:36-44.

12 Sleath B, Blalock S, Covert D, et al. The relationship between glaucoma medication adherence, eye drop technique, and visual field defect severity. Ophthalmology 2011;118:2398-402.

13 Cate $\mathrm{H}$, Bhattacharya $\mathrm{D}$, Clark $\mathrm{A}$, et al. Improving adherence to glaucoma medication: a randomised controlled trial of a patientcentred intervention (the Norwich adherence glaucoma study). BMC Ophthalmol 2014;14:1-10.

14 Fiscella R. The effect of an educational intervention on adherence. 24, 2018.

15 World Health Organization. World report on vision executive summary 36, 2019: 1-12. https://www.who.int/news-room/detail/08-10-2019who-launches-first-world-report-on-vision

16 Ung C, Murakami Y, Zhang E, et al. The association between compliance with recommended follow-up and glaucomatous disease severity in a County hospital population. Am J Ophthalmol 2013:156:362-9.

17 Kotecha A, Fernandes S, Bunce C, et al. Avoidable sight loss from glaucoma: is it unavoidable? Br J Ophthalmol 2012;96:816-20.

18 Traverso CE, Walt JG, Kelly SP, et al. Direct costs of glaucoma and severity of the disease: a multinational long term study of resource utilisation in Europe. Br J Ophthalmol 2005;89:1245-9.

19 Lee PPet al. A multicenter, retrospective pilot study of resource use and costs associated with severity of disease in glaucoma. Arch Ophthal 2006;124:12-19.

20 Lee PP, Kelly SP, Mills RP, et al. Glaucoma in the United States and Europe: predicting costs and surgical rates based upon stage of disease. J Glaucoma 2007:16:471-8.

21 Pezzullo L, Streatfeild J, Simkiss P, et al. The economic impact of sight loss and blindness in the UK adult population. BMC Health Serv Res 2018;18:1-13.

22 Haymes SA, Leblanc RP, Nicolela MT, et al. Risk of falls and motor vehicle collisions in glaucoma. Invest Ophthalmol Vis Sci 2007;48:1149-55.

23 McKean-Cowdin R, Wang Y, Wu J, et al. Impact of visual field loss on health-related quality of life in glaucoma: the Los Angeles Latino eye study. Ophthalmology 2008;115:941-9.

24 Kymes SM, Plotzke MR, Li JZ et al. The increased cost of medical services for people diagnosed with primary open-angle glaucoma: a decision analytic approach. Am J Ophthalmol 2010;150:74-81.

25 Skalicky S, Goldberg I. Depression and quality of life in patients with glaucoma a cross-sectional analysis using the geriatric depression Scale-15. J Glaucoma 2008;17:546-51.

26 Varma R. Succesvolle intuitievelingen. 152, 2012.

27 Lacey J, Cate H, Broadway DC. Barriers to adherence with glaucoma medications: a qualitative research study. Eye 2009;23:924-32.

28 Williams MV, Baker DW, Honig EG, et al. Inadequate literacy is a barrier to asthma knowledge and self-care. Chest 1998;114:1008-15.

29 Kalichman SC, Ramachandran B, Catz S. Adherence to combination antiretroviral therapies in HIV patients of low health literacy. J Gen Intern Med 1999:14:267-73.

30 Schillinger D, Grumbach K, Piette J, et al. Association of health literacy with diabetes outcomes. JAMA 2002;288:475-82.

31 Muir KW, Santiago-Turla C, Stinnett SS, et al. Health literacy and adherence to glaucoma therapy. Am J Ophthalmol 2006;142:223-6.

32 Muir KW, Ventura A, Stinnett SS, et al. The influence of health literacy level on an educational intervention to improve glaucoma medication adherence. Patient Educ Couns 2012;87:160-4.

33 Juzych MS, Randhawa S, Shukairy A, et al. Functional health literacy in patients with glaucoma in urban settings. Arch Ophthalmol 2008;126:718-24.

34 Freedman RB, Jones SK, Lin A, et al. Influence of parental health literacy and dosing responsibility on pediatric glaucoma medication adherence. Arch Ophthalmol 2012;130:306-11.

35 Sleath B, Blalock SJ, Robin A, et al. Development of an instrument to measure glaucoma medication self-efficacy and outcome expectations. Eye 2010;24:624-31.

36 Cook PF, Schmiege SJ, Mansberger SL, et al. Predictors of adherence to glaucoma treatment in a multisite study. Ann Behav Med 2015:49:29-39.

37 Carpenter DM, Blalock SJ, Sayner R, et al. Communication predicts medication self-efficacy in glaucoma patients. Optom Vis Sci 2016:93:731-7.
38 Sleath BL, Blalock SJ, Muir KW, et al. Determinants of self-reported barriers to glaucoma medicine administration and adherence: a multisite study. Ann Pharmacother 2014;48:856-62.

39 Sleath B, Blalock SJ, Carpenter DM, et al. Ophthalmologist-patient communication, self-efficacy, and glaucoma medication adherence. Ophthalmology 2015;122:748-54.

40 Salman M, Andrews C, Heisler M, et al. Psychosocial predictors of glaucoma medication adherence among the support, educate, empower (see) personalized glaucoma coaching pilot study participants. Am J Ophthalmol 2020;216:207-18.

41 Rhee H, Belyea MJ, Brasch J, Ph D, PN P. Family support and asthma outcomes in adolescents: barriers to adherence as a mediator. J Adolesc Heal 2010;47:472-8.

42 Maeda U, Shen B-J, Schwarz ER, et al. Self-Efficacy mediates the associations of social support and depression with treatment adherence in heart failure patients. Int J Behav Med 2013;20:88-96.

43 Wu J-R, Frazier SK, Rayens MK, et al. Medication adherence, social support, and event-free survival in patients with heart failure. Health Psychology 2013;32:637-46.

44 Gu L, Wu S, Zhao S, et al. Association of social support and medication adherence in Chinese patients with type 2 diabetes mellitus. Int J Environ Res Public Health 2017;14:1-11.

45 Bouldin ED, Trivedi RB, Reiber GE, et al. Associations between having an informal caregiver, social support, and self-care among low-income adults with poorly controlled diabetes. Chronic IIIn 2017;13:239-50.

$46 \mathrm{Mi} \mathrm{T}$, Li X, Zhou G, et al. Hiv disclosure to family members and medication adherence: role of social support and self-efficacy. AIDS Behav 2020;24:45-54.

47 Lunnela J, Kääriäinen $\mathrm{M}$, Kyngäs $\mathrm{H}$. The views of compliant glaucoma patients on counselling and social support. Scand $J$ Caring Sci 2010;24:490-8.

48 Papastavrou E, Andreou P, Efstathiou G. Rationing of nursing care and nurse-patient outcomes: a systematic review of quantitative studies. Int J Health Plann Manage 2014;29:3-25.

49 Jones TL, Hamilton P, Murry N. Unfinished nursing care, missed care, and implicitly rationed care: state of the science review. Int $J$ Nurs Stud 2015;52:1121-37.

50 Arksey H, O'Malley L. Scoping studies: towards a methodological framework. Int J Soc Res Methodol 2005;8:19-32.

51 Theofilou P. Translation and cultural adaptation of the multidimensional scale of perceived social support for Greece. Health Psychol Res 2015;3:3-5.

52 Tsilika E, Galanos A, Polykandriotis T, et al. Psychometric properties of the multidimensional scale of perceived social support in Greek nurses. Can J Nurs Res 2019;51:23-30.

53 Abe RY, Wen LdeC, Barker GT, et al. Psychometric properties of the glaucoma treatment compliance assessment tool (GTCAT) in a Brazilian population. J Glaucoma 2018;27:257-65.

54 Barker GT, Cook PF, Schmiege SJ, et al. Psychometric properties of the glaucoma treatment compliance assessment tool in a multicenter trial. Am J Ophthalmol 2015;159:1092-9.

55 Pelikan JM, Röthlin F, Ganahl K. Measuring comprehensive health literacy in general populations: validation of instrument indices and scales of the HLS-EU study. Annu Heal Lit Res Conf 2014.

56 Kondilis BK, Agrafiotis D. The European Health Literacy Survey case of Greece, 2012: 1-14.

57 Sleath B, Blalock SJ, Stone JL, et al. Validation of a short version of the glaucoma medication self-efficacy questionnaire. $\mathrm{Br} J$ Ophthalmol 2012;96:258-62.

58 Mansberger SL, Sheppler CR, McClure TM, et al. Psychometrics of a new questionnaire to assess glaucoma adherence: the glaucoma treatment compliance assessment tool (an American Ophthalmological Society thesis). Trans Am Ophthalmol Soc 2013;111:1-16 http://www.aosonline.org/xactions/2013/1545-6110 v111_p001.pdf\%5Cnhttp://ovidsp.ovid.com/ovidweb.cgi?T=JS\& PAGE $=$ reference $\& D=e m e d 11 \& N E W S=N \& A N=2013509663$

59 Barker GT, Mansberger SL. Psychometric properties of the reduced version of the glaucoma treatment compliance assessment tool (GTCAT). Ophthalmic Epidemiol 2019;26:55-62.

60 Sorensen K, Van den Broucke S, Pelikan J. Measuring health literacy in populations: illuminating the design and development process of HLS-EU-Q. BMC Public Health 2013;13:948.

61 Sørensen K, Pelikan JM, Röthlin F, et al. Health literacy in Europe: comparative results of the European health literacy survey (HLS-EU). Eur J Public Health 2015:25:1053-8.

62 Zimet GD, Dahlem NW, Zimet SG. The multidimensional scale of perceived social support the multidimensional scale of perceived social support. J Pers Assess 1988;52:37-41.

63 Teh WL, Shahwan S, Abdin E, et al. Confirmatory factor analysis and measurement invariance of the multidimensional scale of perceived 
social support in young psychiatric and Non-Psychiatric Asians. Ann Acad Med Singap 2019;48:314-20.

64 Adamczyk K. Development and validation of the Polish-language version of the multidimensional scale of perceived social support (MSPSS). Rev Int Psychol Soc 2013;26:25-48.

65 Park H, Nguyen T, Park H. Validation of multidimensional scale of perceived social support in middle-aged Korean women with diabetes. Asia Pac J Soc Work Dev 2012;22:202-13.

66 Pan Y, Jones PS. Correlates of perceived social support in Chinese adult child caregivers of parent stroke survivors. J Nurs Res 2017;25:383-91.

67 Laksmita OD, Chung M-H, Liao Y-M, et al. Multidimensional scale of perceived social support in Indonesian adolescent disaster survivors: a psychometric evaluation. PLoS One 2020;15:1-12.

68 Denis A, Callahan S, Bouvard M. Evaluation of the French version of the multidimensional scale of perceived social support during the postpartum period. Matern Child Health J 2015;19:1245-51.

69 Ekbäck M, Benzein E, Lindberg M, et al. The Swedish version of the multidimensional scale of perceived social support (MSPSS)--a psychometric evaluation study in women with hirsutism and nursing students. Health Qual Life Outcomes 2013;11:168.

$70 \mathrm{Ng} \mathrm{CG}$, Amer Siddiq AN, Aida SA, et al. Validation of the Malay version of the multidimensional scale of perceived social support (MSPSS-M) among a group of medical students in faculty of medicine, University Malaya. Asian J Psychiatr 2010;3:3-6.

71 Behling O, Law K. Translating questionnaires and other research instruments: problems and solutions. Thousand Oaks, CA,USA: Sage Publications Inc, 2000.

72 Hilton A, Skrutkowski M. Translating instruments into other languages: development and testing processes. Cancer Nurs 2002;25:1-7.
73 Beck CT, Bernal H, Froman RD. Methods to document semantic equivalence of a translated scale. Res Nurs Health 2003;26:64-73.

74 Cha E-S, Kim KH, Erlen JA. Translation of scales in cross-cultural research: issues and techniques. J Adv Nurs 2007:58:386-95.

75 Radhakrishna RB. Tips for developing and testing questionnaires/ instruments. J Ext2007;45.

76 Johanson GA, Brooks GP. Initial scale development: sample size for pilot studies. Educ Psychol Meas 2010;70:394-400.

77 Bentler PM, Chou CP. Practical issues in structural modeling. Sociol Methods Res 1987;16:78-117.

78 Flynn LR, Pearcy D. Four subtle SINS in scale development: some suggestions for strengthening the current paradigm. Int J Mark Res 2001;43:1-14.

79 Jayasekara RS. Focus groups in nursing research: methodological perspectives. Nurs Outlook 2012;60:411-6.

80 Calder BJ. Focus groups and the nature of qualitative marketing research. J Mark Res 1977;14:353-64.

81 Howard E, Hubelbank J, Moore P. Employer evaluation of graduates: use of the focus group. Nurse Educ 1989;14:38-41.

$82 \mathrm{CW} R$, Webb C. Focus groups as a research method: a critique of some aspects of their use in nursing research, 2001.

83 Forman J, Damschroder L. Empirical Methods for Bioethics : A Primer Qualitative Content Analysis Article information . . Elsevier, 2008: 11. 39-62.

84 Fetters MD, Curry LA, Creswell JW. Achieving integration in mixed methods Designs-Principles and practices. Health Serv Res 2013;48:2134-56.

85 Ivankova N V, Creswell JW, Stick SL. Using Mixed-Methods Sequential Explanatory Design : From Theory to Practice. , 2006: 18, 3-20. 